\title{
I ndicadores nutricionais e políticas públicas
}

SEmír A M IS MARTIn S Á LVAR ES D OMEn E

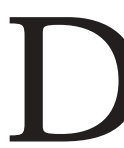

ESDE A REALIZAÇão da Conferência M undial sobre Alimentação, em 1974, organizada pela FAO - Food and Agriculture O rganization, órgão integrante da O rganização das $\mathrm{N}$ ações U nidas - O N U , os governos participantes comprometeram-se a dedicar esforços para garantir o direito inalienável de todo homem, mulher ou criança estar livre do risco da fome e da desnutrição para o desenvolvimento pleno de suas faculdades físicas e mentais. Q uase trinta anos depois, dados apresentados no Segundo Fórum M undial de Alimentação em 2002 indicam que a cada ano o número de desnutridos cai oito milhões. A pesar de parecer muito, a dimensão da fome no mundo é de tal gravidade que, para que em 2015 se alcance a metade do número de desnutridos, esta taxa de redução deve ser de pelo menos 22 milhões por ano. Estes dados demonstram que todas as medidas tomadas até agora resultaram em pequeno impacto.

As razões para a pequena efetividade das iniciativas podem ser diversas. D o ponto de vista do planejamento em saúde, a implantação de um sistema que preveja o diagnóstico, para adequado mapeamento da situação e das necessidades alimentares da população, dos mecanismos e das estruturas já disponíveis para ações de intervenção, em uma perspectiva de médio e longo prazo, depende do estabelecimento de políticas públicas concebidas em uma perspectiva de continuidade, e com ampla participação da sociedade e da comunidade acadêmica.

Exemplo disso se dá na discussão sobre Segurança Alimentar, que tem orientado a agenda de governos e organizações sociais preocupadas com questões relacionadas à suficiência de suprimento de alimentos, e ampliação de sua concepção a partir do conceito de segurança nutricional. Estudos de Vigilância $\mathrm{N}$ utricional e Alimentar contribuem orientando sobre as demandas nutricionais mais importantes de cada grupo social e criando possibilidades de intervenção efetivas. D esta forma, garante-se não só 0 acesso aos alimentos, mas também 0 suprimento suficiente de nutrientes e energia, o que dá adequada dimensão à proposta. 0 conceito sobre segurança nutricional e sua articulação com a segurança al imentar exige a confluência de várias especialidades, o que é, naturalmente, mais complexo (Lobstein, 2002), mas possibilita a ampliação da temática, com a inclusão das situações de risco derivadas de inadequações al imentares não exclusivamente determinadas por carência. A epidemia da obesidade e as elevadas taxas de doenças crônicas não transmissíveis são preocupações importantes em Saúde Pública, e acometem cada vez mais indivíduos de todos os estratos 
sociais. Estudo realizado em Campinas demonstrou a ocorrência de situações aparentemente paradoxais, em que se observa déficit nutricional ou excesso de peso em moradores de bolsões de pobreza (D omene e col., 1999).

As questões relativas à Segurança $N$ utricional foram tema de discussão do G rupo Consultivo para América Latina e Caribe em seu encontro preparatório para o Fórum M undial de Alimentação de 1996 (World Food Summit, 1996), e foram também abordadas pela Associação N orte-americana de N utricionistas (Olson e Holben, 2002).

N o Brasil, a alteração da estrutura socioeconômica da população vem sendo acompanhada da melhoria dos indicadores de desnutrição, conforme dados obtidos nasúltimas décadas ( $M$ onteiro e Conde, 2000). A pesar disso, a insuficiência alimentar ainda é preocupante, e mostra forte associação com renda e escolaridade ( $M$ arins e Almeida, 2002). Em São Paulo, a renda familiar dobrou entre os anos de 1980 e 1990, o número de famílias com baixo poder aquisitivo caiu $50 \%$, e a escolaridade materna cresceu em 1,5 anos. Contudo, a tradução dos indicadores nutricionais e de saúde, em um país com dimensões territoriais tão grandes e diversidades regionais tão marcantes, encobre realidades locais que apresentam particularidades, com ilhas de riqueza e a continuidade de situações de risco em bolsões de pobreza, observando-se claramente a interferência do fator renda quando os dados são desagregados.

M esmo em nações desenvolvidas, a questão pode assumir contornos preocupantes, a partir do entendimento de que situações de insegurança alimentar e nutricional não se restringem à falta de alimentos, e podem afetar negativamente o consumo e a saúde. N osEU A, cerca de $10 \%$ dos domicílios, que representam uma população estimada em 33 milhões de pessoas segundo dados de 2000, estavam expostos a situações de insegurança alimentar (O Ison e H olben, 2002). $\mathrm{N}$ a Europa, em regiões com elevado nível de renda, dados do Seneca, um dos mais relevantes estudos sobre saúde e nutrição já realizados, indicaram a necessidade de acompanhar situações de insegurança alimentar entre idosos, apesar da diminuição dos casos de desnutrição (de Groot e van Staveren, 2002).

Parece ser crescente o consenso em torno da associação entre desequilíbrio alimentar e as principais doenças com relevância em Saúde Pública (Szostak, 1994), o que reforça a importância dos estudos sobre consumo alimentar como parte de uma política de vigilância (Solomons e Valdes-Ramos, 2002). A padronização de técnicas que garantam sensibilidade e especificidade é, contudo, ainda um desafio em estudos populacionais de consumo, como constatado por pesquisadores em diferentes regiões do globo (Szostak, 1994, Robinson e col, 2001, Kaluski e col., 2002).

M uitas das iniciativas de implantação de um programa de Segurança Alimentar e N utricional enfrentam dificuldades muito parecidas, e o conhecimento destas dificuldades pode ser útil para orientar a formulação de políticas na área. São comuns situações de escassez de recursos, que levam invariavelmente a realo- 
cações e revisões dos investimentos inicialmente previstos para o setor saúde. O utra situação que compromete a condução de políticas na área de alimentação e nutrição decorre de desarticulação das informações sobre os indicadores nutricionais, que pode determinar ausência de investimentos em projetos prioritários ou, eventualmente, duplicação de gastos. Soma-se a isto a baixa capacidade de incorporação dos resultados decorrentes dos financiamentos para a reorganização e o planejamento continuado de ações.

A abordagem da questão merece, portanto, a articulação de ações relacionadas a diferentes áreas, como a edu cação, o saneamento e acesso aos serviços de saúde. Para ilustrar como algumas iniciativas são efetivas, pode-se citar as ações promotoras do aleitamento materno para o combate à desnutrição: dados de Almeida e colaboradores (1999), trabal hando com crianças de $\mathrm{N}$ iterói, reforçaram que 0 aleitamento está fortemente associado com escolaridade da mãe, e propôs que é possível reduzir em $40 \%$ os baixos indicadores de comprometimento de altura com medidas de incentivo à lactação materna. 0 estímulo ao al eitamento como estratégia de combate à desnutrição, contudo, em regiões de pobreza extrema, é pouco efetivo para a recuperação dos indicadores nutricionais, embora contribua para diminuir as taxas de mortalidade (Briend e Bari, 1989). E stas situações demonstram inequivocamente como a academia pode colaborar para otimizar o planejamento das ações de saúde, com vistas a seu direcionamento a objetivos específicos, e com isto causar o impacto necessário sobre as demandas sociais.

O país passa por um momento estratégico para a reorganização das ações em nutrição, notadamente influenciado pelo debate em torno do Programa Fome Zero e pelo envolvimento da sociedade, ingredientes básicos para o estabelecimento de um plano de sucesso. Resta contudo ampliar a participação da academia, que não deve se omitir da reflexão, do debate, da formulação de propostas e do acompanhamento das ações.

\section{Bibliografia}

ALM EIDA, R. M.; DE M ARIN S, V. M . e VALLE, J. "Breastfeeding, Socio-economic Conditions and Nutritional Status of Children Younger than 12 M onths in Brazil". A nn Trop Paediatr 1999, set., 19(3), pp. 257-262.

BRIEN D, A. e BARI, A. "Breastfeeding I mproves Survival, but not Nutritional Status, of 12-35 M onths Old Children in Rural Bangladesh". Eur J Clin Nutr 1989, set., 43(9), pp. 603-608.

DE GROOT, C.P. eVAN STAVEREN, W. A. "U ndernutrition in the European Seneca Studies". Clin Geriatr Med 2002, nov., 18(4), pp. 699-708, vi.

KALUSKI, D. N.; OPHIR, E. e AMEDE, T. "Food Security and Nutrition - The Ethiopian Case for Action". Public H ealth N utr 2002, jun., 5(3), pp. 373-381.

LOBSTEIN , T. "Food Policies: A Threat to H ealth?" Proc N utr Soc 2002, nov., 61(4), pp. 579-585. 
MARINS, V. M. e ALMEIDA, R. M. “Undernutrition Prevalence and Social Determinants in Children Aged 0-59 M onths". N iterói, Brasil. A nn H um Biol 2002, nov.-dez., 29(6), pp. 609-618.

MONTEIRO, C. A. e CONDE, W. L. "Secular Trends in Malnutrition and O besity among Children in the City of Sao Paulo, Brazil (1974-1996)". R evista de Saúde Pública 2000, dez., 34(6 Suppl), pp. 52-61.

MONTEIRO, C. A. edE FREITAS, I. C. "Evolution of Socioeconomic D eterminants of $\mathrm{H}$ ealth in Childhood in the City of Sao Paulo, Brazil (1984-1996)". Revista de Saúde Pública 2000, dez., 34(6 Suppl), pp. 8-12.

OLSON, C. M. e HOLBEN, D. H. "Position of the American D ietetic Association: Domestic Food and N utrition Security". J Am Diet A ssoc 2002, dez.,102(12), pp. 1840-1847.

SO LO M O N S, N. W. e VALDES-RAM OS, R. "D ietary A ssessment Tools for D eveloping Countries for U se in M ulti-centric, Collaborative Protocols". Public H ealth N utr 2002, dez., 5(6A), pp. 955-968.

SZOSTAK, W. B. "The N eed for I mproved M ethods of D iet A ssessment for D eveloping and M onitoring Food Policy in Eastern Europe". A m J Clin N utr 1994, jan., 59(1 Suppl), pp. 273S-274S.

World Food Summit. R eport of the R egional N GO Consultation for Latin A merica and the Caribbean on the World Food Summit. Asunción, Paraguay, 1996.

R esu mo - D urante a realização da Conferência M undial sobre Alimentação, em 1974, os governos participantes defenderam o direito inalienável de todo homem, mulher ou criança estar livre do risco da fome e da desnutrição para o desenvolvimento de suas faculdades físicas e mentais. Q uase trinta anos depois, os presentes ao Segundo Fórum M undial de Alimentação, realizado em 1996, comprometeram-se com o conceito de Segurança Alimentar para todos e com a erradicação da fome, reduzindo-se o número de desnutridos à metade até 2015. A pesar dos esforços dispendidos, a persistir a atual taxa de redução, esta meta não será atingida, a menos que se estabeleça a Segurança Alimentar e Nutricional como política pública, especialmente em regiões com pobreza. A escolha do méto do mais adequado para medida de consumo de alimentos e de estado nutricional, e a avaliação dos indicadores mais adequados só será viabilizada por meio da abordagem ampla da questão. O s indicadores adotados em saúde pública podem mascarar realidades particulares atrás de valores médios, especialmente em regiões de pobreza, levando à falsas interpretações sobre estado nutricional e consumo de alimentos. Com este entendimento, o estabelecimento de políticas públicas para a Segurança Alimentar e N utricional depende de uma ação coordenada entre estado, sociedade, academia e o setor produtivo, a fim de que estabeleça um plano efetivo de transformação da realidade.

A вSTRACT - During the World Food Conference in 1974, governments had proclaimed that "every man, woman and child has the inalienable right to be free from hunger and malnutrition in order to develop their physical and mental faculties." Almost thirty years later, participants of the World Food Summit held in 1996, pledged a common commitment to achieving food security for all, and to eradicate hunger, by reducing the number of undernourished people to half until 2015. Despite the efforts, at the average 
annual rate of reduction, this goal will not be attained, unless Food and Nutrition Security is adopted at a policy level, mainly under circumstances of poverty. To choose a suitable method to measure food consumption and nutritional status, and to test adequate risk indicators, a participatory and comprehensive approach is needed. Because of social discrepancies, the commonly assumed median public health risk indicators are not always valid in developing countries, since they can lead to misleading interpretation about nutritional status or food consumption observed in poor areas. To the extent that this assumption is correct, an effective screening mechanism is part of a national food policy for Food and N utrition Security, and should be oriented by the efforts of governments, community, universities and the private sector, to reinforce an alliance to promote a coordinated action.

Semíramis M artins Á Ivares D omene é professora titular da Faculdade de $\mathrm{N}$ utrição da Pontifícia U niversidade C atólica de Campinas (PU C-Campinas).

Recebido e aceito para publicação em 2 de junho de 2003. 